\title{
Transformation of Maritime Education into Distance Online Education
}

\author{
๑ Selçuk Nas \\ Dokuz Eylül University Maritime Faculty, Department of Maritime Education and Training, İzmir, Turkey \\ Keywords \\ Maritime education, Distance online education, Simonline solution
}

We have started to experience a rapid transformation in maritime education, which has traditional principles and methodologies. The forcing of environmental factors and development in communication technologies have a remarkable role in this transformation. An environmental factor that has a large contribution in this change is the excessive demand for trained human power in maritime industry, which has increased during the pandemic. This increase was owing to the reluctance of seafarers to go to the ship during the pandemic because of concerns of not being able to return to their homes at the end of their contracts. However, educational institutions that remained closed during the pandemic were insufficient to meet the trained human power supply needed by the maritime industry. Despite being equipped with large capitals, the inability of these institutions to meet these needs has made their education systems and methodologies questionable. As a result, the maritime education system had to initiate its own transformation.

The transformation that started in maritime education has met some resistance. Although the education of seafarers is conducted under certain standards such as International Convention on Standards of Training, Certification and Watchkeeping for Seafarers (STCW), the tendency to preserve its traditional structure and principles has always been strong. Doyen trainers in the traditional mindset argue that the face-to-face structure of maritime vocational education should be preserved. On one hand, ignoring traditionalists is not possible, especially concerning the attitude, behaviour development, and safety culture training of seafarers. On the other hand, discussions on distance education for developing technical skills continued before the pandemic. During the pandemic, the technological infrastructure of maritime education could be delivered to students who were isolated at home, which led to the transformation in traditional education methods. Towards the end of the COVID-19 pandemic process, the simulator-supported training infrastructure in maritime education started to be delivered to students in their homes with the developments in communication technologies and its usage has started in the service trainings on board ships.

With these developments in technology, the transformation in traditional education methodologies has become inevitable. Applications of distance online education methodologies that have recently started to develop and tried in maritime education systems have given us the opportunity to compare traditional methods with new ones. For this, the "Simonline Solution" results at Dokuz Eylül University Maritime Faculty were examined for advanced simulator-supported training given in the undergraduate seafarer's education, especially in the last years. In addition, the suitability of a curriculum aimed at team learning and skill development for distance online education was investigated using an advanced methodological approach in simulator-supported trainings. Considering the Simonline Solution results, if the distance online simulator supported trainings are conducted with the appropriate infrastructure, technology, methodology, resources, and guidance, the following advantages over the traditional method have been determined:

- 24/7 access to simulator systems by student teams.

- Teams can plan their own simulator run times.

Address for Correspondence: Selçuk Nas, Dokuz Eylül University Maritime Faculty, Department of Maritime Education and Training, İzmir, Turkey

E-mail: snas@deu.edu.tr

ORCID ID: orcid.org/0000-0001-5053-4594

${ }^{\circ}$ Copyright 2021 by the Journal of ETA Maritime Science published by UCTEA Chamber of Marine Engineers 
- Opportunities provided by technology to validate acquired skills.

- Possibility of repeating the given tasks until the team succeeds.

- Awareness of being a team and helping each other among students.

- Benefit for the development of nontechnical skills

- Each team's simulation experience can be followed by other teams

- Ability to self-assess the team's skills and knowledge

- Increasing students' interest in the lesson.

The obligations and necessity are the only factors that break down prejudices and initiate transformations. From this point of view, the pandemic has provided an opportunity to try many untried things in human history with the help of technology. It destroyed prejudices and lit the fire that started many changes in maritime education. The International Maritime Organization (IMO), which is the global standard-setting authority of maritime education, is late in taking initiatives or undertaking a regulatory role in these matters.

Finally, we are pleased to introduce the JEMS 9 (2) to our valued followers. There are valuable and endeavoured studies in this issue of the journal. We hope that these studies will contribute to the maritime industry. I would like to mention my gratitude to authors who sent their valuable studies for this issue, to our reviewers, editorial board, section editors, and associate editors who provide quality publications by following our publication policies diligently. I would also like to express my thanks to LookUs Scientific and Galenos Publishing House who spent great efforts in the preparation of this issue.

Sincerely yours,

Prof. Dr. Selçuk NAS

Editor-in-Chief 